\title{
Polyline Drawings with Topological Constraints
}

\section{Emilio Di Giacomo}

Università degli Studi di Perugia, Perugia, Italy

emilio.digiacomo@unipg.it

(D) https://orcid.org/0000-0002-9794-1928

\section{Peter Eades}

University of Sydney, Sydney, Australia

peter.d.eades@gmail.com

\section{Giuseppe Liotta}

Università degli Studi di Perugia, Perugia, Italy giuseppe.liotta@unipg.it

(D) https://orcid.org/0000-0002-2886-9694

\section{Henk Meijer}

University College Roosevelt, Middelburg, The Netherlands

h.meijer@ucr.nl

\section{Fabrizio Montecchiani}

Università degli Studi di Perugia, Perugia, Italy

fabrizio.montecchiani@unipg.it

(iD) https://orcid.org/0000-0002-0543-8912

\begin{abstract}
Let $G$ be a simple topological graph and let $\Gamma$ be a polyline drawing of $G$. We say that $\Gamma$ partially preserves the topology of $G$ if it has the same external boundary, the same rotation system, and the same set of crossings as $G$. Drawing $\Gamma$ fully preserves the topology of $G$ if the planarization of $G$ and the planarization of $\Gamma$ have the same planar embedding. We show that if the set of crossingfree edges of $G$ forms a connected spanning subgraph, then $G$ admits a polyline drawing that partially preserves its topology and that has curve complexity at most three (i.e., at most three bends per edge). If, however, the set of crossing-free edges of $G$ is not a connected spanning subgraph, the curve complexity may be $\Omega(\sqrt{n})$. Concerning drawings that fully preserve the topology, we show that if $G$ has skewness $k$, it admits one such drawing with curve complexity at most $2 k$; for skewness- 1 graphs, the curve complexity can be reduced to one, which is a tight bound. We also consider optimal 2-plane graphs and discuss trade-offs between curve complexity and crossing angle resolution of drawings that fully preserve the topology.
\end{abstract}

2012 ACM Subject Classification Mathematics of computing $\rightarrow$ Combinatorics, Mathematics of computing $\rightarrow$ Graph theory, Theory of computation $\rightarrow$ Design and analysis of algorithms

Keywords and phrases Topological graphs, graph drawing, curve complexity, skewness- $k$ graphs, $k$-planar graphs

Digital Object Identifier 10.4230/LIPIcs.ISAAC.2018.39

Related Version A full version of the paper is available at [13], https://arxiv.org/abs/1809. 08111.

Acknowledgements We wish to thank Stephen Wismath for useful discussions.

(c) (i) Emilio Di Giacomo, Peter Eades, Giuseppe Liotta, Henk Meijer, and Fabrizio Montecchiani; licensed under Creative Commons License CC-BY

29th International Symposium on Algorithms and Computation (ISAAC 2018).

Editors: Wen-Lian Hsu, Der-Tsai Lee, and Chung-Shou Liao; Article No. 39; pp. 39:1-39:13

Leibniz International Proceedings in Informatics 


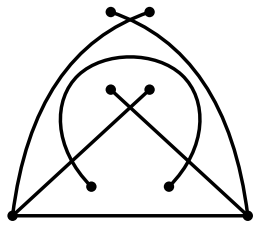

(a)

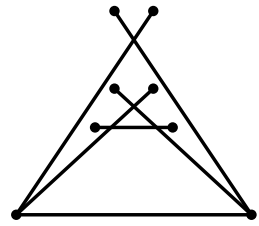

(b)

Figure 1 (a) A topological graph $G$ that requires at least 1 bend in any polyline drawing that fully preserves its topology. (b) A straight-line drawing that partially preserves the topology of $G$.

\section{Introduction}

A fundamental result in graph drawing is the so-called "stretchability theorem" $[12,17,18]$ : Every planar simple topological graph admits a straight-line drawing that preserves its topology. One may ask whether a similar theorem holds for non-planar simple topological graphs. Motivated by the fact that a straight-line drawing may not be possible even for a planar graph plus an edge [10], we allow bends along the edges and measure the quality of the computed drawings in terms of their curve complexity, defined as the maximum number of bends per edge.

Let $G$ be a simple topological graph and let $\Gamma$ be a polyline drawing of $G$. (Note that, by definition of simple topological graph, $G$ has neither multiple edges nor self-loops; see also Section 2 for formal definitions.) Drawing $\Gamma$ fully preserves the topology of $G$ if the planarization of $G$ (i.e., the planar simple topological graph obtained from $G$ by replacing crossings with dummy vertices) and the planarization of $\Gamma$ have the same planar embedding. Eppstein et al. [11] prove the existence of a simple arrangement of $n$ pseudolines that, when drawn with polylines, it requires at least one pseudoline to have $\Omega(n)$ bends. It is not hard to see that the result by Eppstein et al. implies the existence of an $n$-vertex simple topological graph such that any polyline drawing that fully preserves its topology has curve complexity $\Omega(n)$ (see Corollary 2 in Section 2 ). This lower bound naturally suggests two research directions: (i) "Trade" curve complexity for accuracy in the preservation of the topology and (ii) Describe families of simple topological graphs for which polyline drawings that fully preserve their topologies and that have low curve complexity can be computed.

Concerning the first research direction, we consider the following relaxation of topology preserving drawing. A polyline drawing of a simple topological graph $G$ partially preserves the topology of $G$ if it has the same rotation system, the same external boundary, and the same set of crossings as $G$, while it may not preserve the order of the crossings along an edge. It may be worth recalling that some (weaker) notions of topological equivalence between graphs have been already considered in the literature. For example, Kynčl [15, 16] and Aichholzer et al. [1, 2] study weakly isomorphic simple topological graphs: Two simple topological graphs are weakly isomorphic if they have the same set of vertices, the same set of edges, and the same set of edge crossings. Note that a drawing $\Gamma$ that partially preserves the topology of a simple toplogical graph $G$ is weakly isomorphic to $G$ and, in addition, it has the same rotation system and the same external boundary as $G$. Also, Kratochvíl, Lubiw, and Nešetřil [14] define the notion of abstract topological graph as a pair $(G, \chi)$, where $G$ is a graph and $\chi$ is a set of pairs of crossing edges; a strong realization of $G$ is a drawing $\Gamma$ of $G$ such that two edges of $\Gamma$ cross if and only if they belong to $\chi$. The problem of computing a drawing that partially preserves a topology may be rephrased as the problem of computing a strong realization of an abstract topological graph for which a rotation system and an 
external boundary are given in input. A different relaxation of the topology preservation is studied by Durocher and Mondal, who proved bounds on the curve complexity of drawings that preserve the thickness of the input graph [9].

Concerning the second research direction, we investigate the curve complexity of polyline drawings that fully preserve the topology of meaningful families of beyond-planar graphs, that are families of non-planar graphs for which some crossing configurations are forbidden (see, e.g., $[4,8]$ for surveys and special issues on beyond-planar graph drawing). In particular, we focus on graphs with skewness $k$, i.e., non-planar graphs that can be made planar by removing at most $k$ edges, and on 2-plane graphs, i.e., non-planar graphs for which any edge is crossed at most twice. Note that a characterization of those graphs with skewness one having a straight-line drawing that fully preserves the topology is presented in [10]. Also, all 1-plane graphs (every edge can be crossed at most once) admit a polyline drawing with curve complexity one that fully preserves the topology and such that any crossing angle is $\frac{\pi}{2}$ [6].

Our results can be listed as follows. Let $G$ be a simple topological graph.

- If the subgraph of $G$ formed by the uncrossed edges and all vertices of $G$, called planar skeleton, is connected, then $G$ admits a polyline drawing with curve complexity three that partially preserves its topology. If the planar skeleton is biconnected the curve complexity can be reduced to one, which is worst-case optimal (Section 3).

- For the case that the planar skeleton of $G$ is not connected, we prove that the curve complexity may be $\Omega(\sqrt{n})$ (Section 3 ).

- If $G$ has skewness $k$, then $G$ admits a polyline drawing with curve complexity $2 k$ that fully preserves its topology. When $k=1$, the curve complexity can be reduced to one, which is worst-case optimal (Section 4).

- If $G$ is optimal 2-plane (i.e., it is 2-plane and it has $5 n-10$ edges), then $G$ admits a drawing that fully preserves its topology and with two bends in total, and a drawing that fully preserves its topology, with at most two bends per edge, and with optimal crossing angle resolution. The number of bends per edge can be reduced to one while maintaining the crossing angles arbitrarily close to $\frac{\pi}{2}$ (Section 4 ).

We conclude the introduction with an example about the difference between a drawing that fully preserves and one that partially preserves a given topology. Figure 1a shows a simple topological graph for which every polyline drawing fully preserving its topology has at least one bend on some edge. Figure 1b shows a drawing of the same graph that partially preserve its topology and has no bends.

For space reasons some proofs have been omitted and the corresponding statements are marked with an asterisk $\left(^{*}\right)$. Missing details can be found in [13].

\section{Preliminaries}

A simple topological graph is a drawing of a graph in the plane such that: (i) vertices are distinct points, (ii) edges are Jordan arcs that connect their endvertices and do not pass through other vertices, (iii) any two edges intersect at most once by either making a proper crossing or by sharing a common endvertex, and (iv) no three edges pass through the same crossing. A simple topological graph has neither multiple edges (otherwise there would be two edges intersecting twice), nor self-loops (because the endpoints of a Jordan arc do not coincide). A simple topological graph is planar if no two of its edges cross. A planar simple topological graph $G$ partitions the plane into topological connected regions, called faces of $G$. The unbounded face is called the external face. The planar embedding of a simple planar topological graph $G$ fixes the rotation system of $G$, defined as the clockwise circular order of 


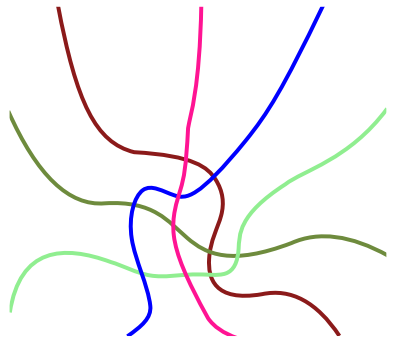

(a)

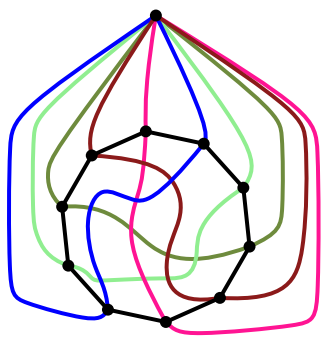

(b)

Figure 2 (a) An arrangement of pseudolines $\mathcal{L}$. (b) The graph $G_{\mathcal{L}}$ associated with $\mathcal{L}$.

the edges around each vertex, and the external face of $G$. The planar skeleton of a simple topological graph $G$ is the subgraph of $G$ that contains all vertices and only the uncrossed edges of $G$. A simple topological graph obtained from $G$ by adding uncrossed edges (possibly none) is called a planar augmentation of $G$.

Let $\mathcal{L}$ be an arrangement of $n$ pseudolines; a polyline realization $\Gamma_{\mathcal{L}}$ of $\mathcal{L}$ represents each pseudoline as a polygonal chain while preserving the topology of $\mathcal{L}$. The curve complexity of $\Gamma_{\mathcal{L}}$ is the maximum number of bends per pseudoline in $\Gamma_{\mathcal{L}}$. The curve complexity of $\mathcal{L}$ is the minimum curve complexity over all polyline realizations of $\mathcal{L}$. The graph associated with $\mathcal{L}$ is a simple topological graph $G_{\mathcal{L}}$ defined as follows. Let $C$ be a circle of sufficiently large radius such that all crossings of $\mathcal{L}$ are inside $C$ and every pseudoline intersects the boundary of $C$ exactly twice. Replace each crossing between $C$ and a pseudoline with a vertex, remove the portions of each pseudoline that are outside $C$, add an apex vertex $v$ outside $C$, and connect $v$ to the vertices of $C$ with crossing-free edges. See Fig. 2 for an example.

- Lemma $1\left(^{*}\right)$. Let $\mathcal{L}$ be an arrangement of $n$ pseudolines and let $G_{\mathcal{L}}$ be the simple topological graph associated with $\mathcal{L}$. Every polyline drawing of $G_{\mathcal{L}}$ that fully preserves its topology has curve complexity $\Omega(f(n))$ if and only if $\mathcal{L}$ has curve complexity $\Omega(f(n))$.

Lemma 1 and the result of Eppstein et al. [11] proving the existence of an arrangement of $n$ pseudolines with curve complexity $\Omega(n)$ imply the following.

- Corollary 2. There exists a simple topological graph with $n$ vertices such that any drawing that fully preserves its topology has curve complexity $\Omega(n)$.

In the next section we study a relaxation of the concept of topology preservation by which we derive constant upper bounds on the curve complexity.

\section{Polyline Drawings that Partially Preserve the Topology}

A polygon $P$ is star-shaped if there exists a set of points, called the kernel of $P$, such that for every point $z$ in this set and for each point $p$ of on the boundary of $P$, the segment $\overline{z p}$ lies entirely within $P$. A simple topological graph is outer if all its vertices are on the external boundary and all the edges of the external boundary are uncrossed. Let $G$ be an outer simple topological graph with $n \geq 3$ vertices and let $P$ be a star-shaped $n$-gon. A drawing $\Gamma$ of $G$ that extends $P$ is such that the $n$ vertices of $G$ are placed at the corners of $P$, and every edge of $G$ is drawn either as a side of $P$ or inside $P$.

- Lemma 3. Let $G$ be an outer simple topological graph with $n \geq 3$ vertices and let $P$ be a star-shaped n-gon. There exists a polyline drawing of $G$ with curve complexity at most one that partially preserves the topology of $G$ and that extends $P$. 


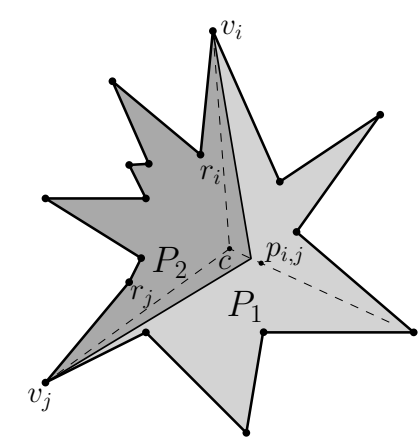

(a)

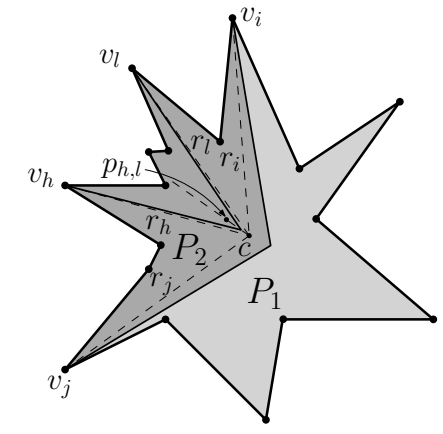

(b)

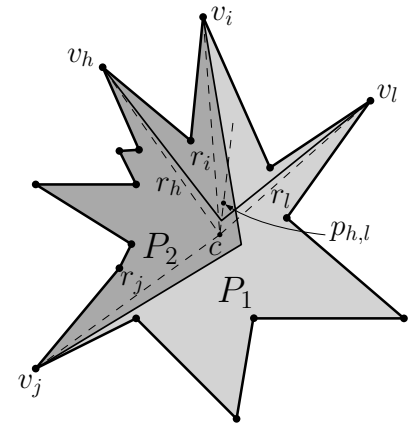

(c)

Figure 3 Illustration for the proof of Lemma 3. (a) The two polygons defined by the addition of edge $\left(v_{i}, v_{j}\right)$. (b) Case 1: $\left(v_{h}, v_{l}\right)$ is contained in $P_{2}$. (c) Case 2: $\left(v_{h}, v_{l}\right)$ intersects $\left(v_{i}, v_{j}\right)$.

Proof. We explain how to compute a drawing with the desired properties for the complete graph $K_{n}$. Clearly a drawing of $G$ can be obtained by removing the missing edges. Identify each vertex of $K_{n}$ with a distinct corner of $P$, and let $\left\{v_{0}, v_{1}, \ldots, v_{n-1}\right\}$ be the $n$ vertices of $K_{n}$ in the clockwise circular order they appear along the boundary of $P$. Note that every edge $\left(v_{i}, v_{i+1}\right)$, for $i=0,1, \ldots, n-1$ (indices taken modulo $n$ ), coincides with a side of $P$ and hence it is drawn as a straight-line segment. We now show how to draw all the edges between vertices at distance greater than one. The distance between two vertices $v_{i}$ and $v_{j}$ is the number of vertices encountered along $P$ when walking clockwise from $v_{i}$ (excluded) to $v_{j}$ (included). We orient each edge $\left(v_{i}, v_{j}\right)$ from $v_{i}$ to $v_{j}$ if the distance between $v_{i}$ and $v_{j}$ is smaller than or equal to the distance between $v_{j}$ and $v_{i}$. The span of an oriented edge $\left(v_{i}, v_{j}\right)$ is equal to the distance between $v_{i}$ and $v_{j}$. We add all oriented edges $\left(v_{i}, v_{j}\right)$ by increasing value of the span. Let $c$ be an interior point of the kernel, for example its centroid. For any pair of vertices $v_{i}$ and $v_{j}$, let $b_{i, j}$ be the bisector of the angle swept by $r_{i}=\overline{c v_{i}}$ when rotated clockwise around $c$ until it overlaps with $r_{j}=\overline{c v_{j}}$. We denote by $\Gamma_{k}$ the drawing after the addition of the first $k \geq 0$ edges and maintain the following invariant for $\Gamma_{k}$.

- For each oriented edge $\left(v_{i}, v_{j}\right)$ not yet in $\Gamma_{k}$, there is a point $p_{i, j}$ on $b_{i, j}$ such that $\left(v_{i}, v_{j}\right)$ can be drawn with a bend at any point of the segment $\sigma_{i, j}=\overline{c p_{i, j}}$ intersecting any edge of $\Gamma_{k}$ at most once (either at a crossing or at a common endpoint).

We will refer to the segment $\sigma_{i, j}$ described in the invariant as the free segment of $\left(v_{i}, v_{j}\right)$. Since $P$ is star-shaped, the invariant holds for $\Gamma_{0}$; in particular the free segment of every $\left(v_{i}, v_{j}\right)$ is the intersection of $b_{i, j}$ with the kernel.

Let $\left(v_{i}, v_{j}\right)$ be the $k$-th edge to be added and assume that the invariant holds for $\Gamma_{k-1}$. We place the bend point of $\left(v_{i}, v_{j}\right)$ at any point of the segment $\sigma_{i, j}$. By the invariant, the resulting edge intersects any other existing edge at most once. We now prove that the invariant is maintained. The drawing of the edge $\left(v_{i}, v_{j}\right)$ divides the polygon $P$ in two sub-polygons (see Fig. 3a). We denote by $P_{1}$ the one that contains the portion of the boundary of $P$ that is traversed when going clockwise from $v_{i}$ to $v_{j}$, and by $P_{2}$ the other one. Notice that the point $c$ is contained in $P_{2}$. Let $\left(v_{h}, v_{l}\right)$ be any oriented edge not in $\Gamma_{k}$. Before the addition of $\left(v_{i}, v_{j}\right)$, by the invariant there was a free segment $\sigma_{h, l}$ for $\left(v_{h}, v_{l}\right)$. By construction, $\left(v_{i}, v_{j}\right)$ intersects $\sigma_{h, l}$ at most once. If $\left(v_{i}, v_{j}\right)$ and $\sigma_{h, l}$ intersect in a point $p$, let $p^{\prime}$ be any point between $c$ and $p$ on $\sigma_{h, l}$ and let $\sigma_{h, l}^{\prime}=\overline{c p^{\prime}}$; if they do not intersect let $\sigma_{h, l}^{\prime}=\sigma_{h, l}$. In both cases $\sigma_{h, l}^{\prime}$ is completely contained in $P_{2}$. We claim that $\sigma_{h, l}^{\prime}$ is a free 


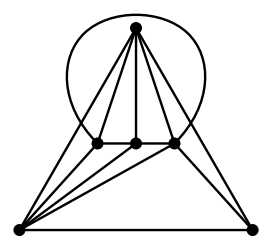

Figure 4 A simple topological graph with a triconnected planar skeleton that does not admit a straight-line drawing that partially preserves its topology.

segment for $\left(v_{h}, v_{l}\right)$. Because of the order used to add the edges, the span of $\left(v_{h}, v_{l}\right)$ is at least the span of $\left(v_{i}, v_{j}\right)$. This implies that $v_{h}$ and $v_{l}$ cannot both belong to $P_{1}$ (as otherwise the span of $\left(v_{h}, v_{l}\right)$ would be smaller than the span of $\left.\left(v_{i}, v_{j}\right)\right)$. We distinguish two cases.

Case 1: Both $v_{h}$ and $v_{l}$ belong to $P_{2}$ (possibly coinciding with $v_{i}$ or $v_{j}$ ). Refer to Fig. 3b. For any point $b$ of $\sigma_{h, l}^{\prime}$, the polyline $\pi$ consisting of the two segments $\overline{v_{h} b}$ and $\overline{b v_{l}}$ is completely contained in $P_{2}$ and therefore does not intersects the edge $\left(v_{i}, v_{j}\right)$ (except possibly at a common end-vertex if $v_{h}$ or $v_{l}$ coincide with $v_{i}$ or $v_{j}$ ). By the invariant, $\pi$ intersects any other existing edge at most once. Thus, $\sigma_{h, l}^{\prime}$ is a free segment.

Case 2: One between $v_{h}$ and $v_{l}$ belongs to $P_{1}$ and the other one belongs to $P_{2}$. Refer to Fig. 3c. For any point $b$ of $\sigma_{h, l}^{\prime}$, the polyline $\pi$ consisting of the two segments $\overline{v_{h} b}$ and $\overline{b v_{l}}$ intersects the edge $\left(v_{i}, v_{j}\right)$ exactly once. By the invariant, $\pi$ intersects any other existing edge at most once. Thus, $\sigma_{h, l}^{\prime}$ is a free segment.

From the argument above we obtain that the final drawing of $K_{n}$ has curve complexity one and extends $P$. By removing the edges of $K_{n}$ not in $G$, we obtain a polyline drawing $\Gamma$ of $G$ with curve complexity one that extends $P$. Moreover, $\Gamma$ partially preserves the topology of $G$. Namely, the circular order of the edges around each vertex and the external boundary are preserved by construction. Furthermore, since $G$ is outer, any two of its edges cross if and only if their four end-vertices appear interleaved when walking along its external boundary. This property is preserved in $\Gamma$, because the order of the vertices along $P$ is the same as the order of the vertices along the external boundary of $G$, and because any two edges cross at most once (either at a crossing or at a common endpoint).

We use Lemma 3 to compute a polyline drawing $\Gamma$ with constant curve complexity for any simple topological graph $G$ that has a biconnected planar skeleton $\sigma(G)$. We triangulate each face of $\sigma(G)$ and compute a straight-line drawing of this triangulation, which contains a drawing of $\sigma(G)$ where each face is a star-shaped polygon. Then, since each edge of $G \backslash \sigma(G)$ is inside one face of $\sigma(G)$, we draw these edges by using Lemma 3. Drawing $\Gamma$ has curve complexity one, which is worst-case optimal, even if the planar skeleton is triconnected (see, e.g., Fig. 4).

- Theorem $4(*)$. Let $G$ be a simple topological graph that admits a planar augmentation whose planar skeleton is biconnected. Then $G$ has a polyline drawing with curve complexity at most one that partially preserves its topology. The curve complexity is worst-case optimal.

If $\sigma(G)$ is connected, we can draw $G$ with three bends per edge.

- Theorem $5\left(^{*}\right)$. Let $G$ be a simple topological graph that admits a planar augmentation whose planar skeleton is connected. Then $G$ has a polyline drawing with curve complexity at most three that partially preserves its topology. 


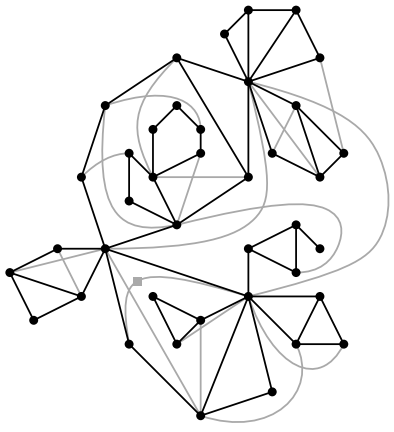

(a)

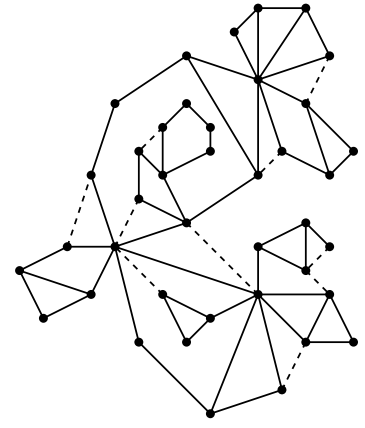

(b)

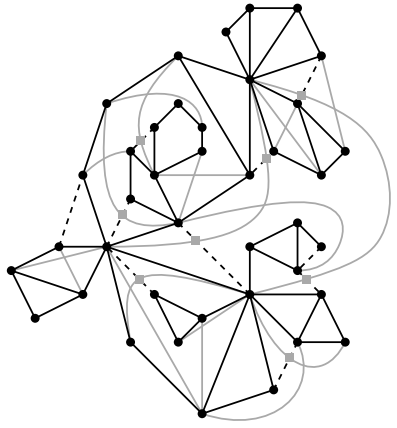

(c)

Figure 5 (a) A simple topological graph $G$. The planar skeleton $\sigma(G)$ of $G$ is shown in black. (b) Augmentation of $\sigma(G)$ to make it biconnected. (c) Augmentation of $G$. Each edge of $G \backslash \sigma(G)$ (in gray) is crossed by the augmenting edges at most twice.

Proof sketch. Let $G^{\prime}$ be a planar augmentation of $G$ whose planar skeleton $\sigma\left(G^{\prime}\right)$ is connected. The idea is to add a set $E^{*}$ of edges to make $\sigma\left(G^{\prime}\right)$ biconnected and then use Theorem 4. For each face $f$ (possibly including the external one) whose boundary contains at least one cutvertex we execute the following procedure. Walk clockwise along the boundary of $f$ and let $v_{0}, v_{1}, v_{2}, \ldots, v_{k}$ be the sequence of vertices in the order they are encountered during this walk, where the vertices that are encountered more than once (i.e., the cutvertices) appear in the sequence only when they are encountered for the first time. For each pair of consecutive vertices $v_{i-1}$ and $v_{i}$ (for $i=1,2, \ldots, k$ ) in the above sequence, if $v_{i-1}$ and $v_{i}$ are not adjacent in $\sigma\left(G^{\prime}\right)$, add to $E^{*}$ the edge $\left(v_{i-1}, v_{i}\right)$. See Fig. $5 \mathrm{a}$ and $5 \mathrm{~b}$ for an example. If we add the edges of $E^{*}$ to $G^{\prime}$ (embedded in the same way with respect to $\sigma\left(G^{\prime}\right)$ ), we obtain a new topological graph such that the edges of $E^{*}$ cross the edges of $G^{\prime} \backslash \sigma(G)$ (see Fig. 5c). Replacing each of the crossings created by the addition of $E^{*}$ with dummy vertices, we obtain a new topological graph $G^{\prime \prime}$ whose planar skeleton is biconnected. By Theorem $4 G^{\prime \prime}$ admits a drawing that partially preserves its topology and such that each edge has at most one bend. Replacing dummy vertices with bends, we obtain a drawing of $G^{\prime}$ that partially preserves its topology. An edge $e$ is split in at most three "pieces" in $G^{\prime \prime}$. The two "pieces" that are incident to the original vertices are not crossed in $G^{\prime \prime}$ and therefore they belong to $\sigma\left(G^{\prime \prime}\right)$ and are drawn without bends. The third "piece" is not in $\sigma\left(G^{\prime \prime}\right)$ and is drawn with at most one bend. Thus, $e$ has at most three bends.

Theorems 4 and 5 show that constant curve complexity is sufficient for drawings that partially preserve the topology of graphs whose planar skeleton is connected. It is worth remarking that a drawing that fully preserves the topology may require $\Omega(n)$ curve complexity even if the planar skeleton is connected. Namely, the planar skeleton of the graphs associated with arrangements of pseudolines is always biconnected and, by Corollary 2, there exists one such graph that has $\Omega(n)$ curve complexity.

One may wonder whether the constant curve complexity bound of Theorems 4 and 5 can be extended to the case on non-connected planar skeletons. This question is answered in the negative by the next theorem.

Theorem $6\left(^{*}\right)$. There exists a simple topological graph with $n$ vertices such that any drawing that partially preserves its topology has curve complexity $\Omega(\sqrt{n})$.

Proof sketch. Let $\mathcal{L}$ be an arrangement of pseudolines and let $G_{\mathcal{L}}$ be the graph associated with $\mathcal{L}$. By Lemma 1 any drawing that fully preserves the topology of $G_{\mathcal{L}}$ cannot have a 


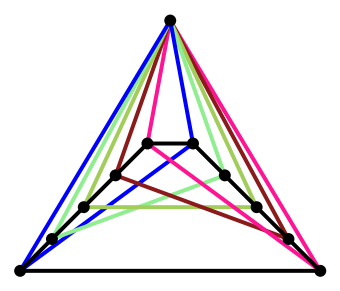

(a)

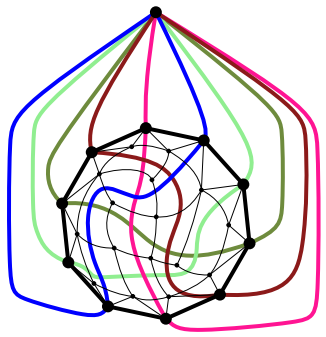

(b)

Figure 6 (a) Straight-line drawing of the graph $G_{\mathcal{L}}$ of Fig. 2b. (b) The graph $\bar{G}_{\mathcal{L}}$ for the arrangement of Fig. $2 \mathrm{a}$.

better curve complexity than $\mathcal{L}$. On the other hand if we only want to partially preserve the topology, $G_{\mathcal{L}}$ can be realized without bends (see Fig. 6a for a straight-line drawing of the graph of Fig. $2 \mathrm{~b}$ ). We now describe how to construct a supergraph $\bar{G}_{\mathcal{L}}$ of $G_{\mathcal{L}}$, such that in any drawing of $\bar{G}_{\mathcal{L}}$ that partially preserves its topology, the topology of the subgraph $G_{\mathcal{L}}$ is fully preserved. Refer to Fig. $6 \mathrm{~b}$ for an illustration concerning the graph of Fig. $2 \mathrm{~b}$. The set $E^{*}$ of crossing edges of $G_{\mathcal{L}}$ forms a set of cells inside the cycle $C$ of $G_{\mathcal{L}}$ (these cells correspond to the internal faces of the planarization of $G_{\mathcal{L}}$ ). For each of these cells, we add a vertex inside the cell and we connect two such vertices if the corresponding cells share a side. For those cells that have as a side an edge $e$ of $C$ we add an edge between the vertex added inside that cell and the two end-vertices of $e$. Let $\bar{G}_{\mathcal{L}}$ be the resulting topological graph and let $\bar{\Gamma}_{\mathcal{L}}$ be a drawing that partially preserves the topology of $\bar{G}_{\mathcal{L}}$. It can be proved that the sub-drawing $\Gamma_{\mathcal{L}}$ of $\bar{\Gamma}_{\mathcal{L}}$ representing $G_{\mathcal{L}}$ fully preserves the topology of $G_{\mathcal{L}}$.

Denote by $\mathcal{L}_{N}$ the arrangement of $N$ pseudolines defined by Eppstein et al. [11]. By the argument above, any polyline drawing that partially preserves the topology of the graph $\bar{G}_{\mathcal{L}_{N}}$ contains a sub-drawing of $G_{\mathcal{L}_{N}}$ that fully preserves its topology and that hence has curve complexity $\Omega(N)$ by Lemma 1. The number of vertices of $G_{\mathcal{L}_{N}}$ is $2 N+1$ and the number of cells is $\Theta\left(N^{2}\right)$. This implies that the number of vertices of $\bar{G}_{\mathcal{L}_{N}}$ is $n=\Theta\left(N^{2}\right)$. Thus, any drawing that partially preserves the topology of $\bar{G}_{\mathcal{L}_{N}}$ has curve complexity $\Omega(N)=\Omega(\sqrt{n})$.

Based on Theorem 6 one may wonder whether $O(\sqrt{n})$ curve complexity is sufficient when the skeleton is not connected. The following theorem states a preliminary result in this direction, extending Theorem 5 to the case that the planar skeleton consists of at most $c$ connected components.

- Theorem $7(*)$. Let $G$ be a simple topological graph that admits a planar augmentation whose planar skeleton has c connected components. Then $G$ has a polyline drawing with curve complexity at most $4 c-1$ that partially preserves its topology.

\section{Polyline Drawings that Fully Preserve the Topology}

In this section we study polyline drawings of constant curve complexity for two meaningful families of beyond-planar graphs. Namely, we consider $k$-skew graphs and 2-plane graphs. A simple topological graph $G=(V, E)$ is $k$-skew if there is a set $F \subseteq E$ of $k$ edges such that $G^{\prime}=(V, E \backslash F)$ does not contain crossings. A simple topological graph is 2-plane if every edge is crossed by at most two other edges. A 2-plane graph with $n$ vertices can have at most $5 n-10$ edges and it is called optimal 2-plane if it has exactly $5 n-10$ edges. We prove that 


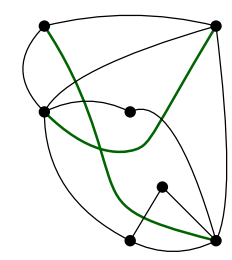

(a)

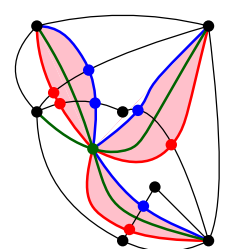

(b)

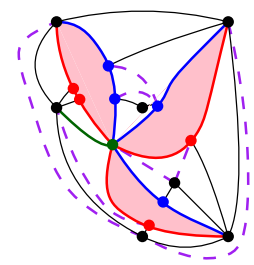

(c)

Figure 7 (a) A topological graph $G$ with a set $F$ of 2 edges (in green) whose deletion makes $G$ planar. (b) A topological graph $G^{\prime \prime}$ formed from $G$ by splitting the edges of $F$ with a dummy vertex and adding a sleeve around each portion of the split edges. (c) The graph obtained by deleting the interior of each sleeve in $G^{\prime \prime}$ and triangulating the graph except for the faces formed by the sleeves.

the graphs belonging to these two families admit a polyline drawing that fully preserves the topology and has constant curve complexity. A tool that we are going to use is the algorithm of Chiba et al. [7] that receives as input a 3-connected plane graph $G$ whose external face has $k \geq 3$ vertices, and a convex polygon $P$ with $k$ corners. The algorithm computes a straight-line drawing $\Gamma$ of $G$ that fully preserves the topology of $G$, it has polygon $P$ as its external face, and all internal faces are convex. Moreover, if three consecutive vertices belong to a same face and are collinear in the computed drawing, we can slightly perturb one of them without destroying the convexity of the other faces. Thus, we can assume that all faces of $\Gamma$ are strictly convex.

We first show that a $k$-skew topological graph admits a polyline drawing that fully preserves the topology of $G$ and has at most $2 k$ bends per edge. The technique is based on an approach that we call the sleeve method and that is illustrated in the following.

The sleeve method. Suppose that $G$ is a topological graph such that the removal of the edge $(s, t)$ makes $G$ without crossings. Let $E_{\chi}$ be the set of edges that cross $(s, t)$ and suppose that $\alpha$ is a crossing between edges $(s, t)$ and $(u, v) \in E_{\chi}$ in $G$. If the clockwise order of the vertices around $\alpha$ is $\langle s, u, t, v\rangle$, then $u$ is a left vertex and $v$ is a right vertex (with respect to the ordered pair $(s, t)$ and the crossing $\alpha)$. We add a "sleeve" around $(s, t)$, as follows. Number the edges of $E_{\chi}=\left\{e_{1}, e_{2}, \ldots, e_{p}\right\}$ in the order of their crossings $\alpha_{1}, \alpha_{2}, \ldots, \alpha_{p}$ along $(s, t)$, so that $e_{i}=\left(u_{i}, v_{i}\right)$ crosses $(s, t)$ at $\alpha_{i}, u_{i}$ is left, and $v_{i}$ is right. We subdivide each edge $\left(u_{i}, v_{i}\right)$ with dummy vertices $u_{i}^{\prime}$ and $v_{i}^{\prime}$ so that the edge $\left(u_{i}, v_{i}\right)$ becomes a path $\left(u_{i}, u_{i}^{\prime}, v_{i}^{\prime}, v_{i}\right)$ with the crossing point $\alpha_{i}$ in between $u_{i}^{\prime}$ and $v_{i}^{\prime}$. Note that after this subdivision, $u_{i}^{\prime}$ is left and $v_{i}^{\prime}$ is right, and $u_{i}$ and $v_{i}$ are neither left nor right. Next we add a path $p_{L}$ that begins at $s$ and visits each of the left dummy vertices $u_{i}^{\prime}$ in the order $u_{1}, u_{2}, \ldots, u_{p}$, and ends at $t$. Similarly we add a path $p_{R}$ that visits $s$, all the right vertices, and then $t$. We call the cycle formed by $p_{L}$ and $p_{R}$ a sleeve. Note that the interior of the sleeve contains the edges $\left(u_{i}^{\prime}, v_{i}^{\prime}\right)$ and the edge $(s, t)$, but no other vertices or edges. The next theorem explains how to draw $k$-skew graphs with curve complexity $2 k$.

- Theorem 8. Every $k$-skew simple topological graph admits a polyline drawing with curve complexity at most $2 k$ that fully preserves its topology.

Proof. Suppose that $G=(V, E)$ is a topological graph and there is a set $F \subseteq E$ of $k$ edges such that deleting all the edges in $F$ from $G$ gives a planar topological graph. An example with $k=2$ is in Fig. 7a. Replace each crossing between a pair of edges in $F$ with a dummy vertex, and let $G^{\prime}$ be the resulting graph. In $G^{\prime}$ there is a set $F^{\prime}$ of edges such that no two 


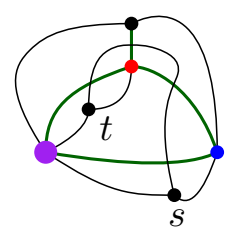

(a)

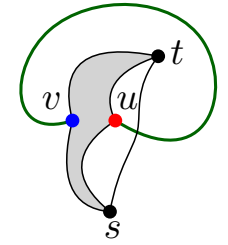

(b)

Figure 8 (a) A 1-skew graph with an inconsistent vertex (larger and purple). (b) A 1-skew graph with an internal inconsistent face (shaded), in which every vertex is consistent.

edges in $F^{\prime}$ cross, and deleting all the edges in $F^{\prime}$ from $G^{\prime}$ gives a planar topological graph. Here $\left|F^{\prime}\right| \leq k+2 c$, where $c$ is the number of crossings between edges in $F$. Also, note that the number of such crossings on each edge in $F$ is at most $k-1$. Now add a sleeve around each edge $(s, t) \in F^{\prime}$ using the sleeve method, and let $G^{\prime \prime}$ be the resulting graph (see Fig. 7b). Note that two such sleeves do not share any edge, and they share at most one vertex. Delete the interior of each sleeve in $G^{\prime \prime}$ to give a planar topological graph $G^{\prime \prime \prime}$. Note that each sleeve of $G^{\prime \prime}$ gives a face of $G^{\prime \prime \prime}$. Now triangulate $G^{\prime \prime \prime}$ except for the faces of $G^{\prime \prime \prime}$ formed by the sleeves (see Fig. 7c).

The resulting graph $G^{i v}$ is triconnected by Barnette's Theorem [3], since two faces share at most one edge or at most one vertex. We can construct a planar drawing $\Gamma^{i v}$ of $G^{i v}$ using the convex drawing algorithm of Chiba et al. [7]. Each face of $\Gamma^{i v}$ is convex, including each face that comes from a sleeve. Drawing the edges of $G^{\prime \prime}$ inside each sleeve as straight-line segments gives a straight-line drawing of $G^{\prime \prime}$. Deleting the dummy edges of the sleeves, and replacing the dummy vertices of the sleeves by bends, we have a polyline drawing $\Gamma$ of $G$ that fully preserves the embedding of $G$. The only bends are (1) at the crossing points between edges of $F$, and (2) at the dummy vertices of the sleeves. Let $e$ be an edge of $G$. If $e \in E \backslash F$, then $e$ crosses at most $k$ edges (those in $F$ ) and each of these crossings creates two dummy vertices in a sleeve of $G^{\prime \prime}$, thus resulting in $2 k$ bends. If $e \in F$, then it has bends at the crossings with other edges of $F$, which are at most $k-1$.

By Theorem 8 we can draw a 1-skew topological graph with two bends per edge. We now prove that these graphs can be drawn using only one bend per edge. To this aim we first recall some results from [10]. We say that a vertex is inconsistent with respect to the edge $(s, t)$ if it is both left and right with respect to $(s, t)$, and consistent otherwise. For example, the graph in Fig. 8a has an inconsistent vertex. Observe that in a straight-line drawing of a topological graph, an inconsistent vertex would have to be both left and right of the straight line through $s$ and $t$. This gives the following necessary condition.

- Lemma 9 ([10]). A 1-skew simple topological graph with an inconsistent vertex has no straight-line drawing that fully preserves its topology.

Without additional assumptions, the converse of Lemma 9 is false. For an example, consider Fig. 8b; this graph has no straight-line drawing, even though all vertices are consistent. The problem is that the internal face $(s, u, t, v)$ has both left and right vertices; as such, this face is inconsistent. To explore the converse of Lemma 9, we can assume that the topological graph is maximal 1-skew (that is, no edge can be added while retaining the property of being 1-skew). Namely, it has been proven that every 1-skew simple topological graph $G$ with no inconsistent vertices can be augmented with dummy edges so that the resulting graph has no inconsistent vertices, it is maximal 1-skew, and it fully preserves the 


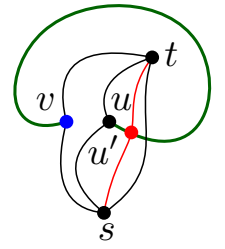

(a)

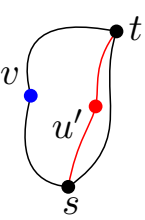

(b)

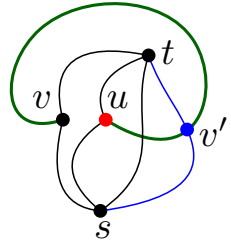

(c)

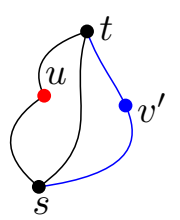

(d)

Figure 9 (a) A left half-sleeve is added to the graph $G$ in Fig. 8(b) to form $G^{* L}$. (b) $G_{L R}^{* L}$ has an internal inconsistent face. (c) A right half-sleeve is added to the graph $G$ in Fig. 8(b) to form $G^{* R}$. (d) $G_{L R}^{* R}$ has no internal inconsistent face.

topology of its subgraph $G$ [10] . Note that both the simple topological graphs in Fig. 8 are maximal 1-skew. We denote the set of left (resp. right) vertices of a 1-skew topological graph $G$ by $V_{L}$ (resp. $V_{R}$ ), the subgraph of $G$ induced by $V_{L} \cup\{s, t\}$ (resp. $V_{R} \cup\{s, t\}$ ) by $G_{L}$ (resp. $G_{R}$ ), the union of $G_{L}$ and $G_{R}$ by $G_{L R}$. Note that $G_{L}$ and $G_{R}$ are induced subgraphs, but $G_{L R}$ is not necessarily induced as a subgraph of $G$. The following is proved in [10].

- Lemma 10 ([10]). Let $G$ be a maximal 1-skew graph with all vertices consistent. Then:

(a) $G_{L R}$ has exactly one inconsistent face, and this face contains both s and t; and

(b) G has a straight-line drawing that fully preserves its topology if and only if the inconsistent face of $G_{L R}$ is the external face (of $G_{L R}$ ).

Let $(s, t)$ be the edge of $G$ whose removal makes $G$ planar. It is clear that after adding a sleeve around edge $(s, t)$, the conditions of Lemma 10 are satisfied and thus, we can compute a straight-line drawing, which after removing the dummy vertices of the sleeve, gives rise to a drawing with at most two bends per edge. To prove that one bend per edge suffices, we need a more subtle argument.

- Theorem $11\left(^{*}\right)$. Every 1-skew simple topological graph admits a polyline drawing with curve complexity at most one that fully preserves its topology. The curve complexity is worst-case optimal.

Proof sketch. Instead of placing a sleeve around the edge $(s, t)$, we use a "half-sleeve", as follows. Again let $E_{\chi}$ be the set of edges that cross $(s, t)$. We 1-subdivide each edge $(u, v) \in E_{\chi}$ with a dummy vertex on the left side of the crossing between $(u, v)$ and $(s, t)$, then add a path $p_{L}$ that begins at $s$ and visits each of the left dummy vertices in the order that their incident edges cross $(s, t)$, and ends at $t$. Denote the graph obtained from $G$ by adding this "left half-sleeve" as above by $G^{* L}$. Similarly, we could add a "right half-sleeve" to obtain a topological graph $G^{* R}$. It is clear that every vertex in both $G^{* L}$ and $G^{* R}$ is consistent. Note also that we have only added one dummy vertex on each edge $(u, v) \in E_{\chi}$; we aim to draw each of these edges with only one bend per edge. However, it is not clear that the internal faces of $G_{L R}^{* L}$ and $G_{L R}^{* R}$ are consistent. Consider, for example, the graph $G$ in Fig. 8(b). For this graph, Fig 9 shows $G^{* L}, G^{* R}, G_{L R}^{* L}$ and $G_{L R}^{* R}$. Note that $G_{L R}^{* L}$ has an internal inconsistent face, while $G_{L R}^{* R}$ does not. One can show that at most one of the graphs $G_{L R}^{* L}$ and $G_{L R}^{* R}$ has an internal inconsistent face. Thus, by Lemma 10, one of these two graphs admits a straight-line drawing which becomes a drawing with curve complexity one after the removal of the dummy vertices used to construct the half-sleeve.

We conclude this section with our results about optimal 2-plane graphs. 
- Theorem $12(*)$. Every optimal 2-plane graph has a polyline drawing $\Gamma$ that fully preserves its topology and that has one of the following properties:

(a) $\Gamma$ has two bends in total.

(b) $\Gamma$ has curve complexity one and every crossing angle is at least $\frac{\pi}{2}-\epsilon$, for any $\epsilon>0$.

(c) $\Gamma$ has curve complexity two and every crossing angle is exactly $\frac{\pi}{2}$.

\section{Open Problems}

Theorem 6 proves a lower bound of $\Omega(\sqrt{n})$ on the curve complexity of polyline drawings that partially preserve the topology and that do not have a connected skeleton. It may be worth understanding whether this bound is tight.

Theorem 12 proves that for optimal 2-plane graphs a crossing angle resolution arbitrarily close to $\frac{\pi}{2}$ can be achieved with curve complexity one, while optimal crossing angle of $\frac{\pi}{2}$ is achieved at the expenses of curve complexity two. Can optimal crossing angle resolution and curve complexity one be simultaneously achieved? A positive answer to this question is known if the planar skeleton of the graph is a dodecahedron [5].

Finally, a natural research direction suggested by the research in this paper is to extend the study of the curve complexity of drawings that fully preserve the topology to other families of beyond-planar topological graphs. For example, it would be interesting to understand whether Theorem 12 can be extended to non-optimal 2-plane graphs.

\section{References}

1 Bernardo M. Ábrego, Oswin Aichholzer, Silvia Fernández-Merchant, Thomas Hackl, Jürgen Pammer, Alexander Pilz, Pedro Ramos, Gelasio Salazar, and Birgit Vogtenhuber. All Good Drawings of Small Complete Graphs. In EuroCG 2015, pages 57-60, 2015.

2 Oswin Aichholzer, Thomas Hackl, Alexander Pilz, Gelasio Salazar, and Birgit Vogtenhuber. Deciding monotonicity of good drawings of the complete graph. In $E G C$ 2015, pages 33-36, 2015.

3 David W. Barnette. 2-Connected Spanning Subgraphs of Planar 3-Connected Graphs. J. Combin. Theory Ser. B, 61(2):210-216, 1994.

4 Michael A. Bekos, Michael Kaufmann, and Fabrizio Montecchiani. Guest Editors' Foreword and Overview. J. Graph Algorithms Appl., 22(1):1-10, 2018.

5 Michael A. Bekos, Michael Kaufmann, and Chrysanthi N. Raftopoulou. On Optimal 2- and 3-Planar Graphs. In SOCG 2017, volume 77 of LIPIcs, pages 16:1-16:16. LZI, 2017.

6 Steven Chaplick, Fabian Lipp, Alexander Wolff, and Johannes Zink. 1-Bend RAC Drawings of NIC-Planar Graphs in Quadratic Area. In GD 2018. Springer, To appear.

7 Norishige Chiba, Kazunori Onoguchi, and Takao Nishizeki. Drawing plane graphs nicely. Acta Inform., 22(2):187-201, 1985.

8 Walter Didimo, Giuseppe Liotta, and Fabrizio Montecchiani. A Survey on Graph Drawing Beyond Planarity. CoRR, abs/1804.07257, 2018. arXiv:1804.07257.

9 Stephane Durocher and Debajyoti Mondal. Relating Graph Thickness to Planar Layers and Bend Complexity. In ICALP 2016, volume 55 of LIPIcs, pages 10:1-10:13. LZI, 2016.

10 Peter Eades, Seok-Hee Hong, Giuseppe Liotta, Naoki Katoh, and Sheung-Hung Poon. Straight-Line Drawability of a Planar Graph Plus an Edge. In WADS 2015, pages 301-313. Springer, 2015.

11 David Eppstein, Mereke van Garderen, Bettina Speckmann, and Torsten Ueckerdt. ConvexArc Drawings of Pseudolines. CoRR, abs/1601.06865, 2016. arXiv:1601.06865.

12 István Fáry. On straight line representations of planar graphs. Acta Univ. Szeged. Sect. Sci. Math., 11:229-233, 1948. 
13 Emilio Di Giacomo, Petere Eades, Giuseppe Liotta, Henk Meijer, and Fabrizio Montecchiani. Polyline Drawings with Topological Constraints. CoRR, abs/1809.08111, 2018. arXiv: 1809.08111.

14 Jan Kratochvíl, Anna Lubiw, and Jaroslav Nešetřil. Noncrossing Subgraphs in Topological Layouts. SIAM J. Discrete Math., 4(2):223-244, 1991.

15 Jan Kynčl. Simple Realizability of Complete Abstract Topological Graphs in P. Discrete Comput. Geom., 45(3):383-399, 2011.

16 Jan Kynčl. Enumeration of simple complete topological graphs. European Journal of Combinatorics, 30(7):1676-1685, 2009.

17 Sherman K. Stein. Convex maps. Proc. Am. Math. Soc., 2(3):464-466, 1951.

18 Klaus Wagner. Bemerkungen zum Vierfarbenproblem. Jahresber. Dtsch. Math. Ver., 46:26$32,1936$. 